\title{
Ab initio derivation of interatomic interactions in transition metals
}

\author{
Z.Gurskii ${ }^{1}$, J.Krawczyk ${ }^{2}$ \\ 1 Institute for Condensed Matter Physics \\ of the National Academy of Sciences of Ukraine, \\ 1 Svientsitskii Str., 79011 Lviv, Ukraine \\ 2 Opole University, 48 Oleska Str., 45-052 Opole, Poland
}

Received August 14, 2000

\begin{abstract}
An approach to the ab-initio calculation of many-body interatomic potentials in transition metals (TM) is developed. It is based on utilizing the local spin density approximation and linear superposition assumption for the density of the valence electrons. The analytic expressions for the spin dependent pair and triplet interatomic interactions are given. The many-body potentials for three particles and four particles are shown to be indirect and to describe interactions of the same spin orientation.
\end{abstract}

Key words: local spin density, total energy functional, many-body potentials

PACS: $34.20 . C f, 71.15 . M b, 71.15 . N c, 71.20 . B e$

\section{Introduction}

Constructing appropriate interatomic potentials is one of the central problems in modern condensed matter physics. It is caused by utilizing these potentials in many physical and material problems. The first-principles and the (semi)empirical methods are the two approaches which dominate this field at present (see review of literature in [1]). In some cases, an adequate theoretical description of metal and alloy properties could not be achieved within the pair interatomic interaction model. That means the many-body interactions play an important role. Transition metals (TM) with partially filled d-bands belong to such objects.

Some many-body empirical models have been advanced to overcome the limitations of the pair potential approximation (see introduction in [2] and papers cited there). Recently, J.Moriarty developed a new theoretical scheme to obtain rigorously triplet and quadruplet angular force interactions within the generalized pseudopotential theory [3]. Another theoretical approach getting the angular forces in TM based on the tight-binding method was proposed in [4]. 
Analyzing the results of [2-4], one can conclude that the three- and four-body interactions play an important role in TM, especially in metals with partially filled d-bands.

Effective interatomic potentials for TM introduced in [2-4] are spin independent. The subject of the present paper is to get these potentials for the case when the spin orientation is included into consideration explicitly. The method proposed in $[1,5]$ for simple metals is generalized here for TM.

\section{The total energy functional within the local spin density approximation}

Let us examine an electron-ion-system of TM taking into account the spin orientation of valence electron density

$$
\rho(\mathbf{r})=\sum_{\sigma} \rho_{\sigma}(\mathbf{r})
$$

where the symbol $\sigma=\uparrow, \downarrow$ distinguishes the up and the down spins. The outer $d$ and $s$-electron shells of TM atoms are treated as valence electrons.

The Kohn-Sham total energy functional of the TM electron-ion system in the spin polarized case has the form [6]

$$
\begin{aligned}
E[\rho]= & \sum_{\sigma} T\left[\rho_{\sigma}\right]+\sum_{\sigma, \sigma^{\prime}} \int V_{\mathrm{ext}}^{\sigma^{\prime}}(\mathbf{r}) \rho_{\sigma}(\mathbf{r}) \mathrm{d} \mathbf{r} \\
& +\frac{1}{2} \sum_{\sigma, \sigma^{\prime}} \int \frac{\rho_{\sigma}(\mathbf{r}) \rho_{\sigma^{\prime}}\left(\mathbf{r}^{\prime}\right)}{\left|\mathbf{r}-\mathbf{r}^{\prime}\right|} \mathrm{d} \mathbf{r} \mathrm{d} \mathbf{r}^{\prime}+\sum_{\sigma} E_{\mathrm{xc}}\left[\rho_{\sigma}\right]+E_{i-i}
\end{aligned}
$$

(all equations are written in atomic units in the present paper). Here, $T\left[\rho_{\sigma}\right]$ and $E_{\mathrm{xc}}\left[\rho_{\sigma}\right]$ are the kinetic and exchange-correlation energy functionals, respectively. The second and the third term in (2.2) describe the energy of the valence electron subsystem in the external field of ions $V_{\text {ext }}^{\sigma}(\mathbf{r})$ and the Hartree energy, correspondingly. The last term in (2.2) is the energy of the ion-ion direct interactions.

The total energy $E[\rho]$ is a universal functional of the electron density, and is obtained by solving the set of the Kohn-Sham equations. The all-electron eigenvalue problem for TM is usually solved by the full-potential-linearized-augmented-planewave method [7].

Suppose the valence electron density $\rho(\mathbf{r})$ is determined within the unrestricted Hartree-Fock method, that is, in the form of (2.1). Let us represent $\rho(\mathbf{r})$ as the linear superposition of contributions from the individual "pseudoions" embedded in the uniform background of density $\rho^{0}$,

$$
\rho_{\sigma}(\mathbf{r})=\rho_{\sigma}^{0}+\sum_{\mathbf{R}} \rho_{i, \sigma}(\mathbf{r}-\mathbf{R}) .
$$

Here,

$$
\sum_{\sigma} \rho_{\sigma}^{0}=\frac{Z_{s}^{*}}{\Omega_{0}}
$$


is the density of the uniform electron distribution, $Z_{s}^{*}$ is an effective ion valency in TM, $\Omega_{0}$ is the atomic volume and $\rho_{i, \sigma}(\mathbf{r}-\mathbf{R})$ is the valence electron density of $\sigma$-spin orientation related to the pseudoion on the site $\mathbf{R}$. Electrons of $s$-symmetry contribute to $\rho_{\sigma}^{0}$ while $d$-electrons contribute to $\rho_{i, \sigma}(\mathbf{r}-\mathbf{R})$. The effective ion valency takes noninteger values in TM owing to the $s-d$ hybridization [8].

The approach proposed is based on two approximations:

i) linear superposition for the valence electron density, see equation (2.3);

ii) local spin density approximation (LSDA) for the exchange-correlation and kinetic energy functionals.

The LSDA for the $E_{\mathrm{xc}}\left[\rho_{\sigma}\right]$ functional in (2.2) reads [6]

$$
E_{\mathrm{xc}}\left[\rho_{\sigma}\right]=\int \mathrm{d} \mathbf{r} \rho_{\sigma}(\mathbf{r}) \varepsilon_{\mathrm{xc}}^{0}\left(\rho_{\sigma}(\mathbf{r})\right)
$$

where $\varepsilon_{\mathrm{xc}}^{0}\left(\rho_{\sigma}(\mathbf{r})\right)$ is the exchange-correlation energy per electron of the interacting electron gas possessing the same density $\rho_{\sigma}(\mathbf{r})$ at point $\mathbf{r}$ as the electron subsystem under investigation.

We represent the known quantity $T_{\sigma}$ for the valence electron kinetic energy extracted from the ab initio band structure calculations in the form of the LSDA, that is,

$$
T\left[\rho_{\sigma}\right]=\int \mathrm{d} \mathbf{r} \rho_{\sigma}(\mathbf{r}) t\left(\rho_{\sigma}(\mathbf{r})\right) .
$$

One can regard equation (2.6) as a constraint on the unknown function $t\left(\rho_{\sigma}(\mathbf{r})\right)$. For explicit expressions for $t\left(\rho_{\sigma}(\mathbf{r})\right)$ see $[1,9]$. As will be shown in the next section, utilizing approximations (i) and (ii) allows one to develop the total energy of TM as a multi-ion interaction expansion

$$
E=E_{0}\left(\Omega_{0}\right)+\frac{1}{2 !} \sum_{\sigma, \sigma^{\prime}} \sum_{\mathbf{R}_{1}, \mathbf{R}_{2}}^{\prime} V_{2}^{\sigma, \sigma^{\prime}}\left(\mathbf{R}_{1}, \mathbf{R}_{2}\right)+\frac{1}{3 !} \sum_{\sigma} \sum_{\mathbf{R}_{1} \ldots \mathbf{R}_{3}}^{\prime} V_{3}^{\sigma}\left(\mathbf{R}_{1}, \mathbf{R}_{2}, \mathbf{R}_{3}\right)+\ldots
$$

where $E_{0}$ represents a volume dependent term including one-ion contributions to $E$, and $V_{2}^{\sigma, \sigma^{\prime}}, V_{3}^{\sigma}$, etc. are the two-particle, three-particle, etc. interatomic potentials which are implicitly volume dependent and explicitly spin dependent. The prime on each $\mathbf{R}$-summation in (2.7) denotes the exclusion of all the self-interaction terms where two indices are equal. It will be shown below that the many-body potentials $V_{n}^{\sigma}\left(\mathbf{R}_{1} \ldots \mathbf{R}_{n}\right), n \geqslant 3$ describe indirect interatomic interactions via electrons of the same spin orientation, that is they are diagonal in the $\sigma$-index.

\section{Spin dependent interatomic potentials}

Let us consider the regular crystal. The electron density $\rho(\mathbf{r}),(2.1)$, as well as the functions $\varepsilon_{\mathrm{xc}}^{0}\left(\rho_{\sigma}(\mathbf{r})\right)$ and $t\left(\rho_{\sigma}(\mathbf{r})\right)$ in (2.5) and (2.6) are periodical because of the crystal periodicity. Therefore, one should consider (2.5) and (2.6) in the region of the 
unit cell only $[1,5]$. The valence electron density $\rho_{\sigma}(\mathbf{r})$, extracted from the ab initio band structure calculations, can be represented in the unit cell region as follows

$$
\rho_{\sigma}(\mathbf{r})=\rho_{p s, \sigma}\left(\mathbf{r}-\mathbf{R}_{1}\right)+\sum_{\mathbf{R} \neq \mathbf{R}_{1}} \rho_{i, \sigma}(\mathbf{r}-\mathbf{R}) \quad \text { for } \quad \mathbf{r} \in \Omega_{0}(1)
$$

where

$$
\rho_{p s, \sigma}\left(\mathbf{r}-\mathbf{R}_{1}\right)=\rho_{\sigma}^{0}+\rho_{i, \sigma}\left(\mathbf{r}-\mathbf{R}_{1}\right)
$$

is the electron density of the $\sigma$-spin orientation related to the pseudoatom on the site $\mathbf{R}_{1}$. The symbol $\mathbf{r} \in \Omega_{0}(1)$ in (3.1) denotes that the running variable $\mathbf{r}$ takes values in the unit cell (the Wigner-Seitz cell) centred on the site $\mathbf{R}_{1}$. The regular TM with one atom per primitive cell is considered in the present paper. The condition of periodicity does not restrict our approach because the potentials $V_{2}^{\sigma \sigma^{\prime}}\left(\mathbf{R}_{1}, \mathbf{R}_{2}\right)$ and $V_{3}^{\sigma}\left(\mathbf{R}_{1}, \mathbf{R}_{2}, \mathbf{R}_{3}\right)$, that will be obtained, are structure independent. The structure dependence of the total energy (2.7) appears through the summation in (2.7) over all $N$ ion positions $[3,5]$.

Let us expand $t\left(\rho_{\sigma}(\mathbf{r})\right)$ and $\varepsilon_{\mathrm{xc}}^{0}\left(\rho_{\sigma}(\mathbf{r})\right)$ in powers of $\rho_{p s, \sigma}\left(\mathbf{r}-\mathbf{R}_{1}\right)$ for $\mathbf{r} \in \Omega_{0}(1)$. For the function $f\left(\rho_{\sigma}(\mathbf{r})\right)=t\left(\rho_{\sigma}(\mathbf{r})\right)$ or $f\left(\rho_{\sigma}(\mathbf{r})\right)=\varepsilon_{\mathrm{xc}}^{0}\left(\rho_{\sigma}(\mathbf{r})\right)$ such an expansion reads

$$
\begin{aligned}
f\left(\rho_{\sigma}(\mathbf{r})\right)= & f\left(\rho_{p s, \sigma}\left(\mathbf{r}-\mathbf{R}_{1}\right)\right)+\frac{\partial f\left(\mathbf{r}-\mathbf{R}_{1}\right)}{\partial \rho_{\sigma}} \sum_{\mathbf{R} \neq \mathbf{R}_{1}} \rho_{i, \sigma}(\mathbf{r}-\mathbf{R}) \\
& +\frac{1}{2 !} \frac{\partial^{2} f\left(\mathbf{r}-\mathbf{R}_{1}\right)}{\partial \rho_{\sigma}^{2}}\left[\sum_{\mathbf{R} \neq \mathbf{R}_{1}} \rho_{i, \sigma}(\mathbf{r}-\mathbf{R})\right]^{2}+\ldots
\end{aligned}
$$

with $\mathbf{r} \in \Omega_{0}(1)$ and

$$
\left.\frac{\partial^{n} f\left(\mathbf{r}-\mathbf{R}_{1}\right)}{\partial \rho_{\sigma}^{n}} \equiv \frac{\partial^{n} f\left(\rho_{\sigma}(\mathbf{r})\right)}{\partial \rho_{\sigma}^{n}(\mathbf{r})}\right|_{\rho_{\sigma}(\mathbf{r})=\rho_{p s, \sigma}\left(\mathbf{r}-\mathbf{R}_{1}\right)} .
$$

To demonstrate a way of treating the exchange-correlation and kinetic energy functionals in terms of the many-body indirect interatomic interactions we consider just equation (2.5) more in detail.

Calculation of the exchange-correlation energy is reduced to the following form

$$
\int_{\Omega} \mathrm{d} \mathbf{r} \rho_{\sigma}(\mathbf{r}) \varepsilon_{\mathrm{xc}}^{0}\left(\rho_{\sigma}(\mathbf{r})\right)=N \int_{\Omega_{0}} \mathrm{~d} \mathbf{r} \rho_{\sigma}(\mathbf{r}) \varepsilon_{\mathrm{xc}}^{0}\left(\rho_{\sigma}(\mathbf{r})\right)
$$

owing to the periodicity of the electron density $\rho_{\sigma}(\mathbf{r})$. Here $\Omega_{0}$ is a volume of a unit cell. In view of (3.1) to (3.3) the right hand side of equation (3.5) is rewritten as follows

$$
\int_{\Omega_{0}(1)} \rho_{\sigma}(\mathbf{r}) \varepsilon_{\mathrm{xc}}^{0}\left(\rho_{\sigma}(\mathbf{r})\right)=\int_{\Omega_{0}(1)} \mathrm{d} \mathbf{r} \rho_{p s, \sigma}\left(\mathbf{r}-\mathbf{R}_{1}\right) \varepsilon_{\mathrm{xc}}^{0}\left[\rho_{p s}\left(\mathbf{r}-\mathbf{R}_{1}\right)\right]
$$




$$
\begin{aligned}
& +\sum_{\mathbf{R}_{2}}^{\prime} \int_{\Omega_{0}(1)} \mathrm{d} \mathbf{r} \rho_{i, \sigma}\left(\mathbf{r}-\mathbf{R}_{1}\right) V_{\mathrm{xc}}^{\sigma}\left(\rho_{p s, \sigma}\left(\mathbf{r}-\mathbf{R}_{1}\right)\right) \\
& +\frac{1}{2} \sum_{\mathbf{R}_{2}}^{\prime} \int_{\Omega_{0}(1)} \mathrm{d} \mathbf{r} \frac{\partial V_{\mathrm{xc}}^{\sigma}\left(\rho_{p s, \sigma}\left(\mathbf{r}-\mathbf{R}_{1}\right)\right)}{\partial \rho_{\sigma}(\mathbf{r})} \rho_{i, \sigma}^{2}\left(\mathbf{r}-\mathbf{R}_{2}\right) \\
& +\sum_{\mathbf{R}_{2}, \mathbf{R}_{3}}^{\prime} \int_{\Omega_{0}(1)} \mathrm{d} \mathbf{r} \frac{\partial V_{\mathrm{xc}}^{\sigma}\left(\rho_{p s, \sigma}\left(\mathbf{r}-\mathbf{R}_{1}\right)\right)}{\partial \rho_{\sigma}} \rho_{i, \sigma}\left(\mathbf{r}-\mathbf{R}_{2}\right) \rho_{i, \sigma}\left(\mathbf{r}-\mathbf{R}_{3}\right)+\ldots
\end{aligned}
$$

Here

$$
V_{\mathrm{xc}}^{\sigma}(\mathbf{r})=\frac{\partial}{\partial \rho_{\sigma}(\mathbf{r})}\left[\rho_{\sigma}(\mathbf{r}) \varepsilon_{\mathrm{xc}}^{0}\left(\rho_{\sigma}(\mathbf{r})\right)\right]
$$

and the symbol $\int_{\Omega_{0}(1)} \ldots$ denotes integration over the unit cell on the site $\mathbf{R}_{1}$, the prime on each summation excludes contributions with $R_{i}=R_{j}(i=1,2,3)$. The procedure to collect in (3.6) the terms of the same order with respect to $\rho_{i, \sigma}(\mathbf{r}-\mathbf{R})$ is a simple one within the given approach. The items up to the order of $\rho_{i, \sigma}^{2}(\mathbf{r}-\mathbf{R})$ only are presented explicitly in (3.6) to avoid saturation of the present paper with too long formulae. They add, see the right hand side of equation (3.6), to the $E_{0}\left(\Omega_{0}\right)$ and to the energy of two-ion and three-ion indirect interactions. One can note from (3.5)(3.7) that the exchange-correlation effects contribute to the indirect interatomic interactions via electrons of the same spin orientation.

The kinetic energy functional is treated in terms of contributions to the indirect interatomic potentials in a similar way [1,5]. Then, using (3.1) to (3.7) and (2.5), (2.6), one can represent the sum $T\left[\rho_{\sigma}\right]+E_{\mathrm{xc}}\left[\rho_{\sigma}\right]$ as follows

$$
\begin{aligned}
& N^{-1}\left(T\left[\rho_{\sigma}\right]+E_{\mathrm{xc}}\left[\rho_{\sigma}\right]\right)=E_{\mathrm{kin}}^{\sigma}\left(\Omega_{0}\right)+E_{\mathrm{xc}}^{\sigma}\left(\Omega_{0}\right) \\
& \quad+\frac{1}{2 !} \sum_{\mathbf{R}_{2}} V_{2, \text { ind }}^{\sigma}\left(\mathbf{R}_{1}, \mathbf{R}_{2}\right)+\frac{1}{3 !} \sum_{\mathbf{R}_{2}, \mathbf{R}_{3}} V_{3, \text { ind }}^{\sigma}\left(\mathbf{R}_{1}, \mathbf{R}_{2}, \mathbf{R}_{3}\right)+\ldots
\end{aligned}
$$

Here,

$$
E_{\mathrm{kin}}^{\sigma}\left(\Omega_{0}\right)=\int_{\left(\Omega_{0}\right)(1)} \mathrm{d} \mathbf{r} \rho_{p s, \sigma}\left(\mathbf{r}-\mathbf{R}_{1}\right) t\left(\rho_{p s, \sigma}\left(\mathbf{r}-\mathbf{R}_{1}\right)\right)
$$

and

$$
E_{\mathrm{xc}}^{\sigma}\left(\Omega_{0}\right)=\int_{\Omega_{0}(1)} \mathrm{d} \mathbf{r} \rho_{p s, \sigma}\left(\mathbf{r}-\mathbf{R}_{1}\right) \varepsilon_{\mathrm{xc}}^{0}\left(\rho_{p s, \sigma}\left(\mathbf{r}-\mathbf{R}_{1}\right)\right)
$$

contribute to $E_{0}\left(\Omega_{0}\right)$ in (2.7). Functions $V_{2 \text {,ind }}^{\sigma}\left(\mathbf{R}_{1}, \mathbf{R}_{2}\right), V_{3 \text {,ind }}^{\sigma}\left(\mathbf{R}_{1}, \mathbf{R}_{2}, \mathbf{R}_{3}\right), \ldots$, describe indirect spin dependent interactions between two, three,..., ions caused by the band structure and exchange-correlation effects.

$$
V_{n, \text { ind }}^{\sigma}\left(\mathbf{R}_{1} \ldots \mathbf{R}_{n}\right)=V_{\text {kin }}^{\sigma}\left(\mathbf{R}_{1} \ldots \mathbf{R}_{n}\right)+V_{\mathrm{xc}}^{\sigma}\left(\mathbf{R}_{1} \ldots \mathbf{R}_{n}\right), \quad n=2,3,4 \ldots
$$


where $V_{\text {kin }}^{\sigma}\left(\mathbf{R}_{1} \ldots \mathbf{R}_{n}\right)$ and $V_{\text {xc }}^{\sigma}\left(\mathbf{R}_{1} \ldots \mathbf{R}_{n}\right)$ are these terms of series for $N^{-1} T\left(\rho_{\sigma}\right)$ and $N^{-1} E_{\mathrm{xc}}\left(\rho_{\sigma}\right)$ which depend on the coordinates of $n$ ions.

Expanding in (2.5) and (2.6) the integrands $\rho_{\sigma}(\mathbf{r}) \varepsilon_{\mathrm{xc}}^{0}\left(\rho_{\sigma}(\mathbf{r})\right)$ and $\rho_{\sigma}(\mathbf{r}) t\left(\rho_{\sigma}(\mathbf{r})\right)$ with respect to $\rho_{i, \sigma}(\mathbf{r}-\mathbf{R})$ up to the $n$-th order, one can derive analytic expressions for the pair and triplet indirect interatomic interactions via electrons in TM, see the right hand side of (3.7) as an example. (Details of calculations for the simple metal case are presented in $[5,10])$.

$$
\begin{aligned}
& \frac{1}{2 !} V_{2, \text { ind }}^{\sigma}\left(\mathbf{R}_{1}, \mathbf{R}_{2}\right)=\sum_{n=0}^{N-2} \frac{1}{(n+1) !} \int_{\Omega_{0}(1)} \mathrm{d} \mathbf{r} \frac{\partial^{n} V_{i n d}^{\sigma}\left(\mathbf{r}-\mathbf{R}_{1}\right)}{\partial \rho_{\sigma}^{n}} \rho_{i, \sigma}^{n+1}\left(\mathbf{r}-\mathbf{R}_{2}\right), \\
& \frac{1}{3 !} V_{3, \text { ind }}^{\sigma}\left(\mathbf{R}_{1}, \mathbf{R}_{2}, \mathbf{R}_{3}\right)=\int_{\Omega_{0}(1)} \mathrm{d} \mathbf{r} \frac{\partial V_{i n d}^{\sigma}\left(\mathbf{r}-\mathbf{R}_{1}\right)}{\partial \rho_{\sigma}} \rho_{i, \sigma}\left(\mathbf{r}-\mathbf{R}_{2}\right) \rho_{i, \sigma}\left(\mathbf{r}-\mathbf{R}_{3}\right) \\
&+\sum_{n=3}^{N} \sum_{m=1}^{n-1} \frac{C_{n}^{m}}{n !} \int_{\Omega_{0}(1)} \mathrm{d} \mathbf{r} \frac{\partial^{n-1} V_{i n d}^{\sigma}\left(\mathbf{r}-\mathbf{R}_{1}\right)}{\partial \rho_{\sigma}^{n-1}} \rho_{i, \sigma}^{n-m}\left(\mathbf{r}-\mathbf{R}_{2}\right) \rho_{i, \sigma}^{m}\left(\mathbf{r}-\mathbf{R}_{3}\right),
\end{aligned}
$$

where $C_{n}^{m}$ are the binomial coefficients. The following notations are introduced in (3.11) to (3.13),

$$
\begin{aligned}
\frac{\partial^{n} V_{i n d}^{\sigma}\left(\mathbf{r}-\mathbf{R}_{1}\right)}{\partial \rho_{\sigma}^{n}} & \left.\equiv \frac{\partial^{n} V_{i n d}^{\sigma}\left(\rho_{\sigma}(\mathbf{r})\right)}{\partial \rho_{\sigma}^{n}(\mathbf{r})}\right|_{\rho_{\sigma}(\mathbf{r})=\rho_{p s, \sigma}\left(\mathbf{r}-\mathbf{R}_{1}\right)}, \\
V_{\text {kin }}^{\sigma}(\mathbf{r}) & =\frac{\partial}{\partial \rho_{\sigma}(\mathbf{r})}\left[\rho_{\sigma}(\mathbf{r}) t\left(\rho_{\sigma}(\mathbf{r})\right)\right] .
\end{aligned}
$$

The first addend in (3.12) and the first term in (3.13) represent irreducible indirect pair and triplet interactions, respectively, in TM. The next addends in (3.12) and (3.13) are contributions to $V_{2 \text {,ind }}^{\sigma}\left(\mathbf{R}_{1}, \mathbf{R}_{2}\right)$ and $V_{3 \text {,ind }}^{\sigma}\left(\mathbf{R}_{1}, \mathbf{R}_{2}, \mathbf{R}_{3}\right)$ arising from the $n$ particle potentials (3.11) when $(n-2)$ or $(n-3)$ indices of ion coordinates coincide, accordingly.

Generalization of equation for the pair total potential [11] on the spin-polarized case of TM does not face any difficulties. Omitting details we present the final result (compare with equation (23) in [1] for $V_{2}\left(\mathbf{R}_{1}, \mathbf{R}_{2}\right)$ in simple metal)

$$
\begin{aligned}
V_{2}^{\sigma \sigma^{\prime}}\left(\mathbf{R}_{1}, \mathbf{R}_{2}\right)= & V_{i-i, \mathrm{dir}}^{\sigma \sigma^{\prime}}\left(\mathbf{R}_{1}, \mathbf{R}_{2}\right)+V_{2, \text { ind }}^{\sigma}\left(\mathbf{R}_{1}, \mathbf{R}_{2}\right) \\
& +\frac{1}{2} \frac{\Omega_{0}^{2}}{(2 \pi)^{3}} \int \mathrm{d} \mathbf{q}\left[V_{\mathrm{ext}}^{\sigma^{\prime}}(q)+V_{\mathrm{H}}^{\sigma^{\prime}}(q)\right] \rho_{i, \sigma}(-\mathbf{q}) \exp \left[\mathrm{iq}\left(\mathbf{R}_{1}-\mathbf{R}_{2}\right)\right] \\
& +\frac{1}{2} \frac{\Omega_{0}^{2}}{(2 \pi)^{3}} \int \mathrm{d} \mathbf{q}\left[V_{\mathrm{ext}}^{\sigma}(q)+V_{\mathrm{H}}^{\sigma}(q)\right] \rho_{i, \sigma^{\prime}}(-\mathbf{q}) \exp \left[\mathrm{iq}\left(\mathbf{R}_{1}-\mathbf{R}_{2}\right)\right]
\end{aligned}
$$

Here $V_{i-i \text {,dir }}^{\sigma \sigma^{\prime}}\left(\mathbf{R}_{1}, \mathbf{R}_{2}\right)$ is the potential of the direct ion-ion interaction, and

$$
V_{\mathrm{H}}^{\sigma}(\mathbf{q})=\frac{4 \pi \rho_{p s, \sigma}(\mathbf{q})}{q^{2}}
$$


$V_{\text {ext }}^{\sigma}(\mathbf{q}), \rho_{i, \sigma}(\mathbf{q})$ and $\rho_{p s, \sigma}(\mathbf{q})$ are the Fourier transforms of $V_{\text {ext }}^{\sigma}(\mathbf{r}), \rho_{i, \sigma}(\mathbf{r})$ and $\rho_{p s, \sigma}(\mathbf{r})$, respectively.

It is seen from (3.16) that just the Coulomb potentials $\left(V_{i i}^{\text {dir }}, V_{\text {ext }}, V_{\mathrm{H}}\right)$ cause interactions between atoms of different spin orientation. The band structure and exchange-correlation effects (see $V_{2 \text {,ind }}^{\sigma}(3.12)$ ) contribute to the effective interatomic potential $V_{2}^{\sigma \sigma}\left(\mathbf{R}_{1}, \mathbf{R}_{2}\right)$ that describes interactions of the same spin orientation.

\section{Discussion}

The focal point of the linear superposition assumption (2.3) and LSDA is an accurate real-space treatment of the TM total energy in terms of well-defined interatomic potentials, see (2.7). The $V_{2}^{\sigma \sigma^{\prime}}, V_{3}^{\sigma}$ etc. potentials entering expansion (2.7) are volume and spin dependent, but explicitly structure independent. Thus, they are rigorously transferable at a given volume to all bulk structures, either ordered or disordered. They can be used for example in computer simulations of TM properties in solid, liquid and amorphous phases.

\section{References}

1. Gurskii Z., Krawczyk J. // Acta Physica Polonica A, 1998, vol. 94, p. 671.

2. Xu W., Adams J.B. // Surface Science, 1994, vol. 301, p. 371.

3. Moriarty J.A. // Phys. Rev. B., 1990, vol. 42, p. 1609; Phys. Rev. B., 1994, vol. 49, p. 12431.

4. Carlsson A.E. // Phys. Rev. B., 1991, vol. 44, p. 6590.

5. Gurskii Z., Krawczyk J. // Cond. Matt. Phys., 1999, vol. 2, p. 383.

6. Callaway J., March N.H. // Solid State Physics, 1984, vol. 38, p. 136.

7. Wimmer E., Krakauer H., Weinert M., Freeman A.J. // Phys. Rev. B., 1981, vol. 24, p. 864.

8. Yukhnovskii I.R., Gurskii Z.A. Quantum-Statistical Theory of Disordered Systems. Kyiv, Naukova Dumka, 1991 (in Russian).

9. Ghosh S.K., Deb B.M. // J. Phys. B.: At. Mol. Opt. Phys., 1994, vol. 27, p. 381.

10. Gurskii Z., Krawczyk J. // Metallofiz. Noveishie Tekhnol., 1999, vol. 21, No. 3, p. 10.

11. Gurskii Z., Krawczyk J. // Met. Phys. Adv. Tech., 1998, vol. 16, p. 1335. 


\title{
Визначення із перших принципів міжатомних взаємодій у перехідних металах
}

\author{
3.Гурський ${ }^{1,2}, €$. Кравчик, $^{2}$
}

1 Інститут фізики конденсованих систем НАН України, 79011 Львів, вул. Свєнціцького, 1

2 Університет Ополє, Польща, 45-052 Ополє, вул. Олеска, 48

Отримано 14 серпня 2000 р.

Розвинуто оригінальний підхід обчислення багаточастинкових міжатомних потенціалів у перехідних металах. Він грунтується на використанні наближення локальної густини спінів та припущенні про те, що густину валентних електронів можна представити у вигляді лінійної суперпозиції. Подані явні вирази для парних та потрійних взаємодій, що залежать від орієнтації спіна. Показано, що багаточастинкові потенціали (три-, чотиричастинкові) описують опосередковані міжатомні взаємодії однакової орієнтації спінів.

Ключові слова: локальна густина спінів, функціонал повної енергії, багаточастинкові потенціали

PACS: $34.20 . C f, 71.15 . \mathrm{Mb}, 71.15 . \mathrm{Nc}, 71.20 . \mathrm{Be}$ 